\title{
Rapid Topographical Plasticity of the Visuomotor Spatial Transformation
}

\author{
Juan Fernandez-Ruiz, ${ }^{1,2}$ Rosalinda Diaz, ${ }^{1}$ Pablo Moreno-Briseño, ${ }^{1}$ Aurelio Campos-Romo, ${ }^{1}$ and Rafael 0jeda ${ }^{1}$ \\ ${ }^{1}$ Departamento de Fisiología, Facultad de Medicina, Universidad Nacional Autónoma de México, 04510 México D.F., México, and 2Escuela de Psicología, \\ Universidad Anáhuac, Lomas Anáhuac, 52786, México
}

\begin{abstract}
Information about the world is often encoded in the brain as topographic maps. These internal representations are not always static but can have a dynamic nature, allowing for constant adjustments that depend on factors like experience or injury. Recently, it has been shown that areas involved in visuomotor transformations also show topographical organization. These findings suggest that it could be possible to observe plastic modifications in specific parts of the representation in response to a local perturbation that affects only a part of the space that is represented. Here, we tested this hypothesis using an adaptation paradigm with hemiprisms. Our results suggest that, initially, the system tries to modify the visuomotor transformation in the whole spatial representation; however, if feedback is available from both hemifields, the system can perform specific regional topographical realignments. The results also suggest that access to the rearranged visuomotor transformation is independent of eye position, in contrast with previous studies that found a kind of conditional learning. Also, whereas prism adaptation experiments using ballistic movements do not show intermanual transfer of learning, the topographical modification found here is available to both hands. These results provide strong evidence for rapid topographical plasticity that can modify space transformations between two different modalities.
\end{abstract}

Key words: topographical representation; prism adaptation; motor learning; procedural learning; visuomotor learning; visuomotor transformation

\section{Introduction}

Information about the world from different sensory modalities including vision, audition, and somatosensation (Buonomano and Merzenich, 1998; Knudsen, 2002) is often encoded in the brain as topographic maps. These internal representations are not always static, and they frequently have a dynamic nature that allows for constant adjustments by factors like experience or injury (Recanzone et al., 1992; Weinberger, 1995). Recently, it has been shown that areas involved in visuomotor transformations also show a topographical organization (Andersen et al., 1997; Sereno et al., 2001). A topographical representation in these areas opens the possibility of selective modifications performed by plastic mechanisms in specific parts of the representation, like those that occur in other sensory modalities. If internal representations involved in visuomotor transformations are ruled by similar plastic mechanisms, then they should show regional modifications in response to a local perturbation that affects only a part of the space represented.

Initial attempts to study visuomotor plasticity include earlier prism adaptation studies. Although most of that research focused

Received Sept. 22, 2005; revised Dec. 6, 2005; accepted Jan. 3, 2006.

This work was supported by Universidad Nacional Autónoma de México PAPIIT Grant IN213802. We are grateful to Herb Goltz and Dorothy Pless for revising this manuscript, Vanessa Murray and Silvia Revuelta for testing subjects, and the volunteers participating in this study.

Correspondence should be addressed to Dr. Juan Fernandez-Ruiz, Laboratorio de Neuropsicologia, Departamento de Fisiología, Facultad de Medicina, Universidad Nacional Autónoma de México, A.P. 70-250, C.P. 04510 México D.F., México. E-mail: jf@@servidor.unam.mx.

DOI:10.1523/JNEUROSCI.4023-05.2006

Copyright $\odot 2006$ Society for Neuroscience $\quad$ 0270-6474/06/261986-05\$15.00/0 on perceptual changes induced by optic distortions of the prisms, some experiments specifically addressed the visuomotor problem using hemiprisms (Kohler, 1964; Pick et al., 1969). These studies found visuomotor gaze-contingent adaptations after using hemiprisms for periods ranging from $4 \mathrm{~h}$ to many weeks. Specifically, they found that the system could display two visuomotor mappings, one for targets presented in the direction of the prismatically viewed field and the other for targets presented in the direction of the undistorted field. However, because eye position was always tied to gaze direction during these studies, it was assumed that the eye position was cuing the visuomotor mapping that should be used with or without prism. These studies could not differentiate whether the system was using two visuomotor mappings or one spatially modified mapping. Other studies, however, have specifically shown that the system is capable of accessing multiple visuomotor mappings cued by different stimuli or contexts (Cunningham and Welch, 1994; Ghahramani et al., 1996; Martin et al., 1996).

Behind the postulation of two visuomotor mappings cued by eye position in the hemiprism studies is the assumption that both normal and displaced maps covered the entire spatial representation. This assumption fits with the idea that changes in space perception shift space rigidly (Bedford, 1993). For example, if a subject is asked to point to a specific target using prisms that displace the visual field $10^{\circ}$ to the left, then, after adaptation, the subject will point $10^{\circ}$ to the left of any other space location. There is, however, another possible explanation for the hemiprism results. Instead of generating multiple visuomotor mappings with 


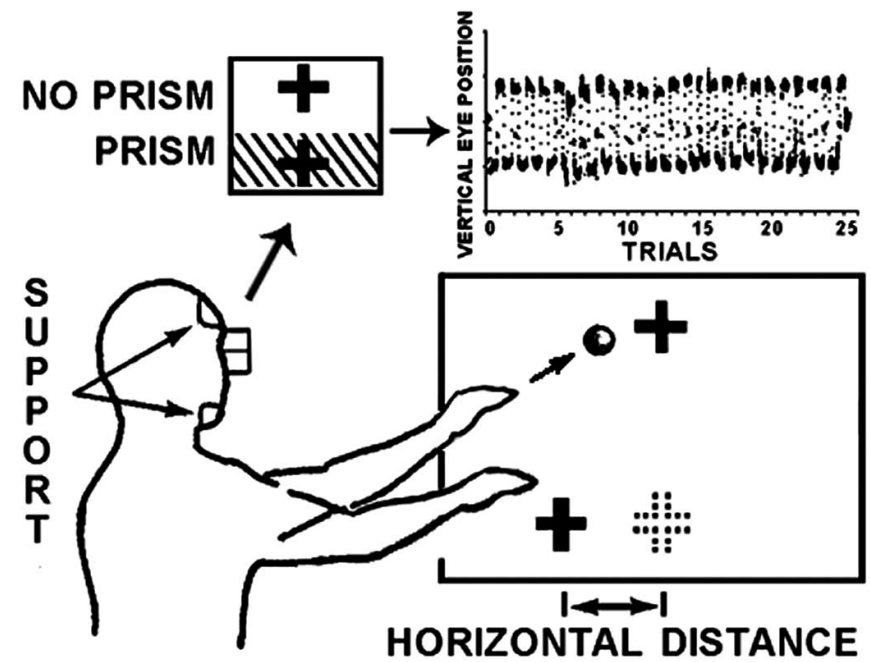

Figure 1. Experimental setup. Using a computer-controlled projector, two targets were presented in an alternating manner $2 \mathrm{~m}$ away from the subject. One target was $45 \mathrm{~cm}$ above and the other was $45 \mathrm{~cm}$ below the subject's eyes. The vertical presentation of the targets was arranged such that they always appeared to be aligned with each other regardless of the introduction of the prisms. With their heads restrained by a chin and forehead support, subjects had to throw clay balls with the right hand to the appearing target. Eye movements were monitored with an optic track device. A typical record of the vertical eye positions while a subject alternated throws is shown.

entire space shifts for different eye positions, one single visuomotor map may be locally modified in a specific part of the representation.

Here, we tested whether the system can modify the visuomotor transformation map in one region of the spatial representation by using a hemiprism adaptation paradigm. If the system can use space-specific visuomotor mappings, it would be compatible with the notion that the system has topographical representations of space (Sereno et al., 2001) and that these topographical representations can be modified regionally without necessarily affecting the whole representation. In contrast, if the system uses a clustered representation of space using a foveation-based signal, or if the plastic mechanisms cannot modulate specific areas of a topographic representation, then the map would be adjusted as a whole, shifting space everywhere (Bedford, 1993; Cohen and Knudsen, 1999; Knudsen, 2002).

\section{Materials and Methods \\ Subjects}

One hundred right-handed, young, healthy university students (one-half men; one-half women) between the ages of 18 and 24 years participated in this study. All subjects were volunteers and were informed about the general nature of the experiment according to the Declaration of Helsinki.

\section{General procedure}

As shown in Figure 1, we tested subjects using a setup that divided the visual field into two hemifields (upper and lower), one without and the other with a prism that produced an optical lateral displacement (but see the description of groups below). Subjects had to alternate throws of clay balls (weight, $10 \mathrm{~g}$ ) toward a $12 \times 12 \mathrm{~cm}$ target (a cross) projected onto a large sheet of parcel paper $2 \mathrm{~m}$ in front of them. The cross was projected using a computer-controlled projector that changed the position of the target every $4 \mathrm{~s}$. For throws in the upper hemifield, the cross was projected $45 \mathrm{~cm}$ above eye height. For throws in the lower hemifield, the cross was projected $45 \mathrm{~cm}$ below eye level. Subjects were instructed to use their right hands, to make all throws overhand, and to throw the balls to the location where they saw the target. The subjects were seated with head restrained by a chin and forehead rest. No directions were given about trunk or shoulder posture. The subjects had an unobstructed view of the target during the entire session; however, they were instructed not to look down at their hand as they collected the next ball from a tray located on a table right next to their bodies. The locations of the ball impacts for each hemifield were plotted sequentially by trial number (abscissa) versus horizontal displacement (in centimeters) from a vertical line passing through the target center (ordinate). Impacts to the left of the target were plotted as negative values, and impacts to the right were plotted as positive values.

\section{Experimental conditions}

The experiments had three conditions as reported previously (Fernandez-Ruiz and Diaz, 1999). In condition PRE, subjects threw balls at the target without any visual displacement. While making their throws during condition PRI, subjects viewed the target through 30 diopter Fresnel 3M Press-on plastic lenses (3M Health Care, St. Paul, MN) that produced a deviation of the space to the right (base left). The prisms were mounted on goggles that were secured by Velcro straps. Subjects wore goggles with prisms encompassing either both or only one hemifield, depending on the group to which they were assigned (see below). The number of throws to the upper or lower hemifield during condition PRI varied for each group (see group descriptions below). Before making throws in the POS condition, goggles were removed from the subjects. The subjects always saw the alternating targets vertically aligned with or without prisms. When the subjects were looking through prisms, the targets were displaced to the left, so the subjects saw them straight ahead.

\section{Groups}

Subjects were divided into six groups (A, B, C, and D: 20 subjects per group; E and F: 10 subjects per group) depending on the PRI characteristics as follows (see Fig. 2).

Group A. Subjects in this group had prisms in both upper and lower visual hemifields. The target, however, only appeared in the lower hemifield. During conditions PRE, PRI, and POS, subjects made 25, 250, and 25 throws, respectively.

Group B. Subjects in this group had prisms in both upper and lower visual hemifields. During each condition, the target alternated between the two hemifields. The subjects made 25, 250, and 25 throws to each hemifield during PRE, PRI, and POS, respectively.

Group C. Subjects in this group had prisms only in the lower hemifield. During each condition, the target alternated between the two hemifields. However, during condition PRI, they threw balls only to the lower hemifield. Therefore, the subjects made 25 throws each to the upper hemifield during PRE and POS, and they made 25, 250, and 25 throws to the lower hemifield during PRE, PRI, and POS, respectively.

Group D. Subjects in this group had prisms only in the lower hemifield. During each condition, the target alternated between the two hemifields. The subjects made 25, 250, and 25 throws to each hemifield during PRE, PRI, and POS, respectively.

Group E. This group was similar to group D, except that during PRI the subjects had to look toward the hemifield opposite to where they saw the target (a circle indicated the position where they should look). They were instructed, however, to keep aiming the balls at the target where the cross appeared.

Group F. This group was similar to group D, except that they made five additional throws with their eyes closed both after PRE and after PRI.

\section{Eye tracking}

The position of the eyes was monitored in every throw by an ISCAN (Burlington, MA) real-time data monitoring system coupled to an RK.426PC Pupil/Corneal Reflection Tracking System that allowed us to do on-site calibrations subject by subject. Although the system tracks eye movements in both vertical and horizontal dimensions, we were interested in the horizontal eye movements that indicated whether subjects were looking to the upper or lower hemifield. The horizontal displacing prism did not affect these measurements. The setup included an infrared illuminator, an eye camera connected to the eye-tracking circuit card in a personal computer that, in turn, fed into an eye monitor.

Preliminary studies were done to position the eye tracker in such a way 


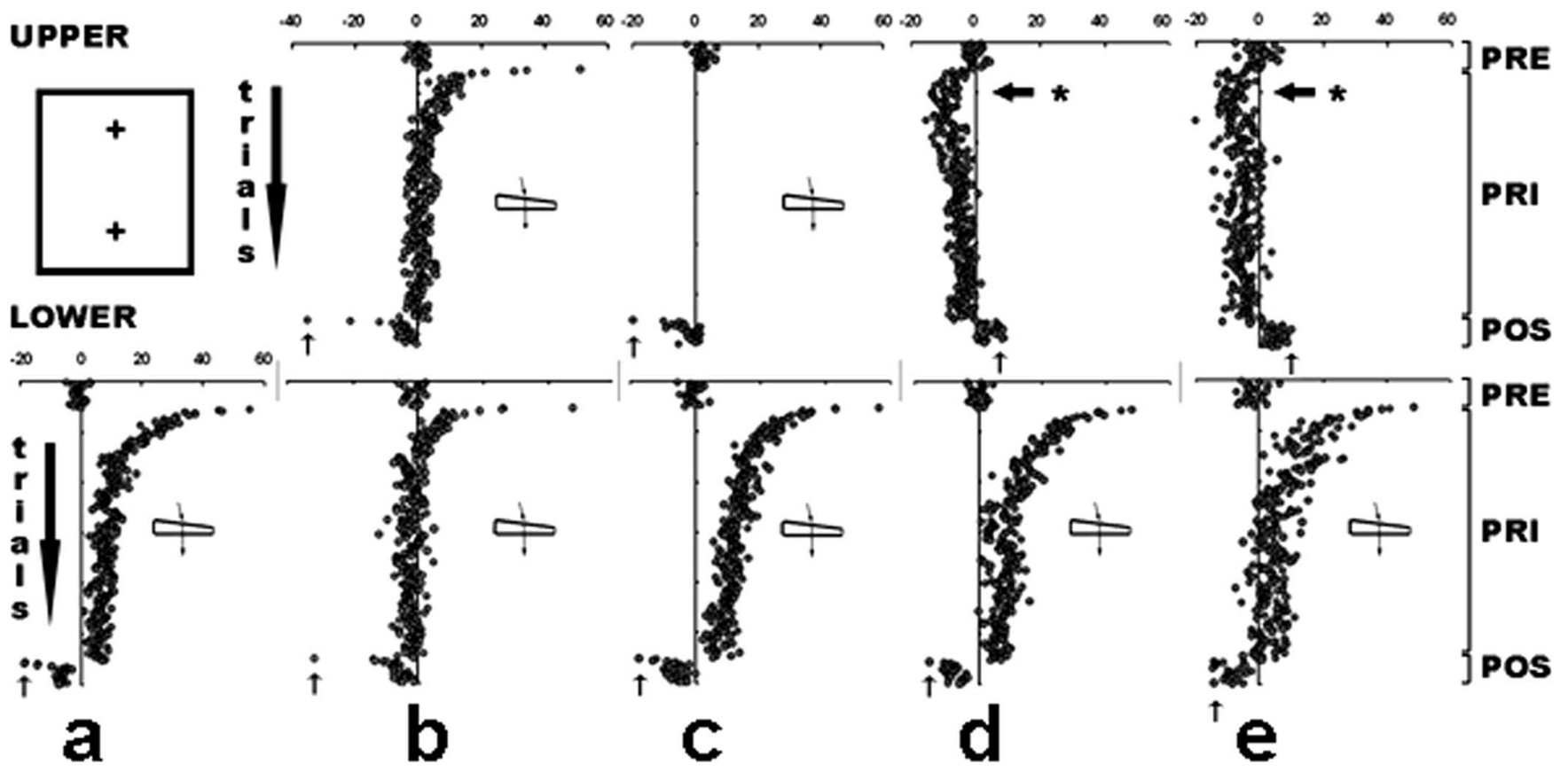

Figure 2. The visuomotor map is affected by plastic mechanisms that try initially to modify the whole spatial representation. If the system is topographically organized and the perturbation affects only a specific area of the map, then the system can show rapid plastic changes that lead to a significant change only in the affected region of the map. Throwing balls in both prism-displaced hemifields ( $\boldsymbol{b}$ ) leads to a faster, more robust adaptation that was followed by a larger aftereffect than throwing only to the lower hemifield (a). Throwing balls only in one prism-displaced hemifield produces significant aftereffects in the untrained area (c). Having one hemifield without and one with prisms ( $\boldsymbol{d}$ and $\boldsymbol{e}$ ) produces an initial deviation on both hemifields (indicated by an asterisk in the upper hemifield). Continuous interactions, however, led to a topographical differentiation between the two hemifields that is eye-position independent (e). The top and bottom graphs represent throws to the upper and lower hemifields, respectively. The horizontal distance to the target (in centimeters) is represented on the $x$-axis, and the trial number is on the $y$-axis. A prism vignette indicates the presence of prisms in that specific condition. The vertical arrows indicate the maximum aftereffect distances.

that there was no interference with the mechanics of the task. The eye tracking showed a clearly detectable difference of eye position while the subjects were looking at the target in either hemifield (Fig. 1).

\section{Measurements}

First, while wearing the prisms, an adaptation measure was obtained by subtracting the horizontal distance between the impact of the ball and the target on the final throw from that on the initial throw of the PRI condition. Second, after removing the prisms at the beginning of the POS condition, an aftereffect measure was defined as the horizontal distance between the impact and target of the first throw. Third, the adaptation rate was measured by the sequence of throws during the PRI condition. For the straight-ahead throws in group F, the measure was the average of the five throws made before and the five throws made after the PRI condition, all of which were made with eyes closed.

\section{Results}

General statistical analyses

Given below are the statistical analyses of the three experimental phases between groups using omnibus ANOVAs. The $p$ values in the rest of Results pertain to the Student-Newman-Keuls (SNK) post hoc analysis described here.

\section{Baseline (PRE)}

An ANOVA test on the baselines found no group differences $(F=$ 1.25 ; $\mathrm{df}=8 ; p=0.21$ ). For all groups, the SNK analysis on the baselines showed no differences between groups (Fig. 2).

\section{Adaptation (PRI)}

The statistical analysis of the adaptation magnitude showed that there were differences between groups $(F=17.076$; $\mathrm{df}=7$; $p<$ 0.01). An SNK showed that the adaptation magnitudes for the upper hemifield in D and $\mathrm{E}$ (where there were no prisms) were similar, but they were different from the rest of the adaptations. No other differences were found among groups (subset for $\alpha=$ $0.05)$. The adaptation rate analysis showed statistical differences between throws $(F=29.664 ; \mathrm{df}=49 ; p<0.01)$ and between throws per group $(F=1.901$; $\mathrm{df}=343 ; p<0.01)$. An SNK revealed three homogeneous subsets of groups: first, groups D and $\mathrm{E}$ in the upper hemifield; second, group B in both hemifields; and third, groups A, C, D, and E in the lower hemifields. The subset for $\alpha$ was $=0.05$.

Additional analysis of the adaptation rate of groups $\mathrm{D}$ and $\mathrm{E}$ for the first 25 throws during PRI in the upper hemifields (without prisms) shows significant differences from their baselines $(F=6.339 ; \mathrm{df}=3 ; p<0.01)$. The SNK indicated two subsets, one for the baselines and one for the upper hemifield throws (Fig. 2, groups D and E, arrows with asterisks).

\section{Aftereffect (POS)}

The aftereffect statistical analysis showed differences between groups $(F=15.401$; $\mathrm{df}=8 ; p<0.01)$. A subsequent SNK revealed three subsets $(\alpha=0.05)$. First, both group B aftereffects were significantly greater than those of the other groups. Second, groups A, C, D, and E in their lower hemifield and group $\mathrm{C}$ in the upper hemifield were both included in one subset. Finally, in the last subset, both $\mathrm{D}$ and $\mathrm{E}$ upper throws were the only ones to exhibit negative aftereffects. For group F, repeated measures ANOVA on the straight-ahead throws made before and after adaptation in both hemifields showed significant differences between groups $(F=23.9$; $\mathrm{df}=7,32 ; p<0.01)$. 


\section{Tendency to make generalized compensations to the whole spatial representation}

Initially, we tested whether the system has a tendency to make generalized compensations to the whole spatial representation. First, we completed control group A (Fig. $2 a$ ), where subjects threw only to the lower hemifield. Then, we added groups B and $\mathrm{C}$ to test plasticity interactions between the two hemifields in the presence or absence of visuomotor behavior in the upper hemifield during the adaptation. Group B (Fig. 2b), which had prisms in both hemifields, showed a faster than normal adaptation in the lower hemifield than control group A $(p<0.001)$, suggesting an active interaction between hemifields. However, if subjects had the opportunity to interact in only one prism-perturbed hemifield, and if they were deprived of interactions in the other hemifield (group C: throws to the upper hemifield only during PRE and POS, but not during the critical PRI phase), then the system generalizes the compensation to the whole spatial representation, showing aftereffects in the lower hemifield that were similar to group $\mathrm{A}(p=0.98)$ and group $\mathrm{B}(p=0.08)$. The most interesting result from this group is that, although they did not make throws to the upper hemifield during PRI, these subjects showed an aftereffect of the same magnitude in both hemifields $(p=1.0)$ (Fig. $2 c$ ). This suggests that the system generalizes the compensation to the whole spatial representation when it is deprived of interactions in one hemifield. These initial findings agree with previous reports suggesting a rigid shift of the visuomotor transformation of the entire spatial representation (Bedford, 1993; Vetter et al., 1999).

\section{The system can sustain topographical plasticity}

Contrary to previous studies that found rigid space shifts (Bedford, 1993), our methodology allowed us to dissociate the axis of displacement from the axis of perturbation-affected areas (Cunningham and Welch, 1994; Ghahramani et al., 1996). The results obtained with this setup suggest that the system can sustain topographical plasticity given enough visuomotor feedback in both hemifields. Group D was designed to test whether the system could adapt to two different conditions, one in each hemifield, by making alternating throws into the two hemifields. Accordingly, group D (Fig. $2 d$ ) made throws into the prism-free and the prismdisplaced hemifields. An analysis of the 25 initial PRI throws in the upper hemifield showed a significant deviation from baseline $(p<0.001)$ (Fig. $2 d$, horizontal arrow) in the same direction as the one observed simultaneously in the lower hemifield. This initial deviation, however, returned toward the baseline level by the second half of the period, although without fully reaching it. Meanwhile, the lower hemifield showed normal adaptation. If aftereffects were different, it would suggest a real change of the internal representation beyond the influence of the prism. The results show aftereffects in opposite directions in the upper and lower hemifields $(p<0.001)$. The aftereffect in the lower hemifield is similar to that observed in groups A and $C$ ( $p=1.0)$, but smaller than group B $(p=0.002)$. The behavior in the upper hemifield of group D suggested, again, that the plastic mechanisms acting on the visuomotor transformation initially affected the whole spatial representation. If this initial trend had maintained its course, it would have supported the hypothesis that the spatial internal representation of the visuomotor transformation had to shift rigidly everywhere, as suggested by the results of groups B and C. However, the trend stopped and reverted toward the baseline, suggesting that the system can distinguish between the two topographic zones. The critical test, however, was to determine the aftereffect magnitudes. Upper and lower hemifield aftereffects were different, suggesting a real change of the internal representation beyond the influence of the prism. These results clearly show that the system can sustain topographical plasticity in a restricted area of the visuomotor transformation spatial representation. Similar findings have been found using a setup that displays virtual images of the position of the effector (Ghahramani et al., 1996).

\section{The hemifield adaptation is not gaze contingent}

Previous long-term studies suggest the possibility that the system develops a new visuomotor representation that is accessed instead of the original one (McGonigle and Flook, 1978; Welch et al., 1993; Martin et al., 1996; Knudsen, 2002). In humans, the formation of new representations requires long periods of training and shows two characteristics: first, the system requires a cue to know when to access it; and second, it does not show aftereffects once the cue is gone (Martin et al., 1996). In previous studies using similar hemiprism approaches (Kohler, 1964; Pick et al., 1969), it was concluded that the eye positions acted as cues instructing the system to switch between internal representations. To evaluate whether we were observing a new internal representation, or a topographical modification of the original, we tested group E, which was similar to $\mathrm{D}$, but the subjects had to make the throws to the target opposite their gaze direction (Fig. 2e). The prediction would be that, if the eye positions were cuing the expression of the newly acquired representation, then an adaptation acquired with the eyes looking down would displace throws to the upper target while looking down and vice versa. The results do not support this hypothesis, because they clearly show an aftereffect in the lower hemifield similar to group $\mathrm{D}(p=1.0)$, although the subjects were inverting their gaze with respect to their throws. Therefore, we concluded that, under these experimental conditions, eye position does not cue the access to the adapted state.

\section{Adaptation mechanism}

A final test to determine whether the system is showing topographical realignments would be to demonstrate transfer to the untrained hand. One way to demonstrate this is to test for intermanual transfer of the proprioceptive component of prism adaptation with a shift test (Redding and Wallace, 2002). Group F followed the same protocol as group D, except that subjects were asked to make five straight-ahead throws with their eyes closed after the initial baseline, and another five similar throws after the adaptation phase with both the trained and the untrained hand. Straight-ahead throws made to the lower hemifield with both right and left hands were different from both upper hemifield and all baseline throws (all significant comparisons were $p<0.01$, and all nonsignificant comparisons were $p=1.0$ ). These results show a significant topographical distribution with either hand (Fig. 3), which is an indication of an intermanual transfer that conserved the topography of the learned realignment. This suggests that the modified internal representation of the visuomotor transformation circuit is available to both hands and that it clearly affects the proprioceptive component of prism adaptation through realignment (Welch et al., 1974; Redding et al., 2005).

\section{Discussion}

These results provide crucial evidence that the visuomotor system can make rapid local plastic changes in response to visual perturbations circumscribed to a specific topographic area. These findings represent a phenomenon different from the multiple visuomotor mappings found after long training periods (McGonigle and Flook, 1978). Whereas the first phenomenon 


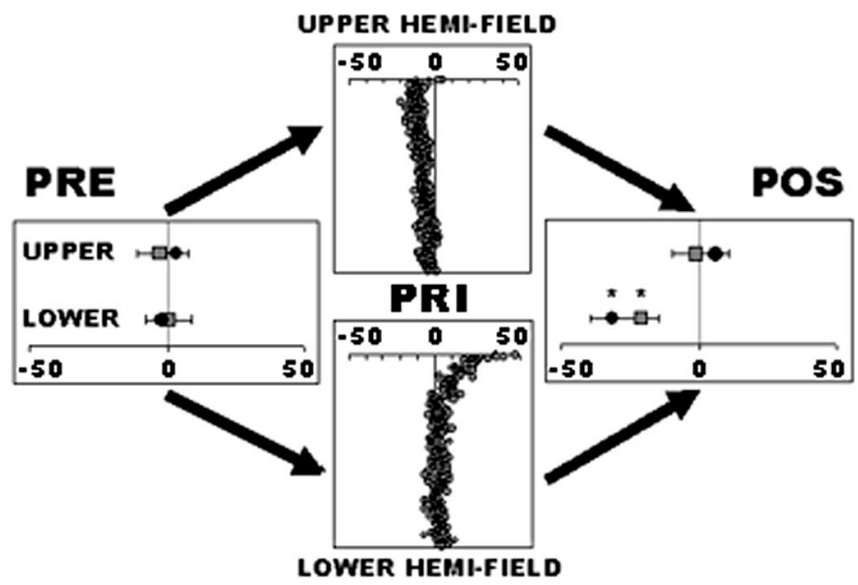

Figure 3. To test whether the plastic process observed is produced by spatial realignment of the visuomotor map, it is necessary to apply a paradigm different from the one used during training. Subjects trained with the right hand in the prism adaptation task display topographical displacements in an eyes-closed, straight-ahead throwing task with both right and left hands. The black circles represent the right hand, and the gray squares are left-hand measures. PRE and POS are straight-ahead throws with eyes closed. PRI shows the right-hand throws during adaptation. ${ }^{*} p<0.01$. Error bars are mean \pm SEM of all subjects.

implies a rapid plastic change in a region of the visuomotor map that is not dependent on cues like eye position, the second one implies the slow generation and storage of new visuomotor transformations that need to be applied throughout the entire map, and that need different cues to be accessed (Kohler, 1964; Welch, 1971; Kravitz and Yaffe, 1972a,b; Welch et al., 1993; Martin et al., 1996; Fernandez-Ruiz et al., 2000).

The topographical plasticity observed here seems to be stimulated by competing input signals arising from different regions of the spatial map. Our results suggest that, in the absence of conflicting inputs, the default setting of the system is a tendency to generalize the adaptation to the entire map (Bedford, 1993; Ghahramani et al., 1996; Vetter et al., 1999). This hypothesis would explain the initial displacement in the prism-free upper hemifields of groups $\mathrm{D}$ and $\mathrm{E}$. If there are conflicting input signals arising from different regions of the map, then the system can make rapid local plastic changes. The mechanisms serving such plastic changes could also act in the alignment between other sensory modalities using fundamental plastic mechanisms found in different topographical rearrangements (Buonomano and Merzenich, 1998).

\section{References}

Andersen RA, Snyder LH, Bradley DC, Xing J (1997) Multimodal representation of space in the posterior parietal cortex and its use in planning movements. Annu Rev Neurosci 20:303-330.
Bedford FL (1993) Perceptual and cognitive spatial learning. J Exp Psychol Hum Percept Perform 19:517-530.

Buonomano DV, Merzenich MM (1998) Cortical plasticity: from synapses to maps. Annu Rev Neurosci 21:149-186.

Cohen YE, Knudsen EI (1999) Maps versus clusters: different representations of auditory space in the midbrain and forebrain. Trends Neurosci 22:128-135.

Cunningham HA, Welch RB (1994) Multiple concurrent visual-motor mappings: implications for models of adaptation. J Exp Psychol Hum Percept Perform 20:987-999.

Fernandez-Ruiz J, Diaz R (1999) Prism adaptation and aftereffect: specifying the properties of a procedural memory system. Learn Mem 6:47-53.

Fernandez-Ruiz J, Hall-Haro C, Diaz R, Mischner J, Vergara P, Lopez-Garcia JC (2000) Learning motor synergies makes use of information on muscular load. Learn Mem 7:193-198.

Ghahramani Z, Wolpert DM, Jordan MI (1996) Generalization to local remappings of the visuomotor coordinate transformation. J Neurosci 16:7085-7096.

Knudsen EI (2002) Instructed learning in the auditory localization pathway of the barn owl. Nature 417:322-328.

Kohler I (1964) The formation and transformation of the perceptual world. New York: International Universities Press.

Kravitz JH, Yaffe FL (1972a) Conditioned adaptation to prismatic displacement. Percept Psychophys 11:38-42.

Kravitz JH, Yaffe F (1972b) Conditioned adaptation to prismatic displacement with a tone as the conditional stimulus. Percept Psychophys 12:305-308.

Martin TA, Keating JG, Goodkin HP, Bastian AJ, Thach WT (1996) Throwing while looking through prisms. II. Specificity and storage of multiple gaze-throw calibrations. Brain 119:1199-1211.

McGonigle BO, Flook J (1978) Long-term retention of single and multistate prismatic adaptation by humans. Nature 272:364-366.

Pick Jr HL, Hay JC, Martin R (1969) Adaptation to split-field wedge prism spectacles. J Exp Psychol 80:125-132.

Recanzone GH, Merzenich MM, Jenkins WM, Grajski KA, Dinse HR (1992) Topographic reorganization of the hand representation in cortical area $3 \mathrm{~b}$ owl monkeys trained in a frequency-discrimination task. J Neurophysiol 67:1031-1056.

Redding GM, Wallace B (2002) Strategic calibration and spatial alignment: a model from prism adaptation. J Mot Behav 34:126-138.

Redding GM, Rossetti Y, Wallace B (2005) Applications of prism adaptation: a tutorial in theory and method. Neurosci Biobehav Rev 29:431-444.

Sereno MI, Pitzalis S, Martinez A (2001) Mapping of contralateral space in retinotopic coordinates by a parietal cortical area in humans. Science 294:1350-1354.

Vetter P, Goodbody SJ, Wolpert DM (1999) Evidence for an eye-centered spherical representation of the visuomotor map. J Neurophysiol 81:935-939.

Weinberger NM (1995) Dynamic regulation of receptive fields and maps in the adult sensory cortex. Annu Rev Neurosci 18:129-158.

Welch RB (1971) Discriminative conditioning of prism adaptation. Percept Psychophys 10:90-92.

Welch RB, Choe CS, Heinrich DR (1974) Evidence for a three-component model of prism adaptation. J Exp Psychol 103:700-705.

Welch RB, Bridgeman B, Anand S, Browman KE (1993) Alternating prism exposure causes dual adaptation and generalization to a novel displacement. Percept Psychophys 54:195-204. 